\title{
Applying skin graft sheets transversely to manage burn patients
}

\author{
Ricardo Madiedo', Jorge Luis Gaviria-Castellanos ${ }^{1}$, Alejandro Zapata-Ospina² \\ ${ }^{1}$ Cosmetic and Reconstructive Plastic Surgeon, Burn Unit Simón Bolívar North Subnetwork E.S.E., Bogotá D.C. 110221, Colombia. \\ ${ }^{2}$ Department of Plastic Surgery, University of Sinú, Cartagena 110231, Colombia.
}

Correspondence to: Dr. Ricardo Madiedo, Cosmetic and Reconstructive Plastic Surgeon, Burn Unit Simón Bolívar North Subnetwork E.S.E., Bogotá D.C. 110221, Colombia. E-mail: madiedor@hotmail.com

\begin{abstract}
How to cite this article: Madiedo R, Gaviria-Castellanos JL, Zapata-Ospina A. Applying skin graft sheets transversely to manage burn patients. Plast Aesthet Res 2018;5:40. http://dx.doi.org/10.20517/2347-9264.2018.40
\end{abstract}

Received: 4 Jun 2018 First Decision: 14 Aug 2018 Revised: 30 Aug 2018 Accepted: 4 Sep 2018 Published: 11 Oct 2018

Science Editor: Raúl González-García Copy Editor: Cui Yu Production Editor: Zhong-Yu Guo

\begin{abstract}
Aim: The aim of this study is to determine whether the split-thickness grafts should be applied to the treatment of burn patients, parallel to the relaxed skin tension lines as described by Kraissl, which would help scarring and reduce the development of abnormal scars.

Methods: A descriptive longitudinal case series study was conducted at the Burn Unit of Health Services Simón Bolívar North Subnetwork E.S.E. of the Secretariat of Health in Bogotá, Colombia, from 1 Jan 2016 to 31 Dec 2017. A total of 138 burn patients exhibiting deep partial- or full-thickness burns involving different body regions, except for the face, were included. Burns required split-thickness skin grafts for definite wound coverage, and these were applied transversely following relaxed skin tension lines. Results were assessed according to the Vancouver Scar Scale (VSS) during a 12-month follow-up. Within this period, function was assessed by the rehabilitation unit, after which physical and functional activities were determined and splinting was implemented, as per rehabilitation protocols, to control and improve functional and physical outcomes.
\end{abstract}

Results: Based on the VSS, most patients (134) had a mean score of 5 (range: 3-7), resulting in clinically acceptable scars with no functional limitations.

Conclusion: Applying split-thickness skin grafts to cover deep partial- or full-thickness burns along relaxed skin tension lines, such as Kraissl's lines, seems to reduce wound tension force, which constitutes one of the most predominant factors contributing to the development of abnormal scars and functional sequelae.

Keywords: Split-thickness grafts, burns, relaxed skin tension lines, hypertrophic scar, keloid, contracture

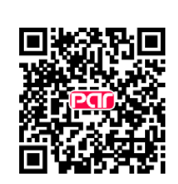




\section{INTRODUCTION}

Burns are considered a public health problem worldwide due to their associated high mortality rates as well as their great morbidity incidence and functional impairment ${ }^{[1-3]}$. Severe scars and late contractures occurring after the placement of split-thickness autografts during treatment persist as some of the most relevant and frustrating sequelae in patients with deep burns ${ }^{[4,5]}$. Not only do they result in deformities and functional impairments, but they may also affect the patient's esthetic appearance, which is distinguishable in burn patients ${ }^{[6,7]}$. These sequelae usually cause prolonged disability, job abandonment, and high costs to the health system $^{[8,9]}$.

Generally, multiple reconstructive interventions are required, including Z-plasties, contracture release, skin grafts, flaps, tissue expanders, skin substitutes, and a great variety of auxiliary treatments, in the attempt to improve and preclude recurrence ${ }^{[10-13]}$. Furthermore, lengthened rehabilitation interventions are required in addition to multiple complementary elements, such as Lycra garments, silicone gel sheets, thermoplastic masks, prolonged immobilization, orthosis, moisturizing lotions, lubricants, corticosteroid injections, and even radiotherapy. Varying results obtained are poor and disheartening in some cases ${ }^{[14-16]}$.

In the literature, it is widely reported that the use of full-thickness skin grafts exhibit better texture and color, and they significantly reduce the occurrence of contractures and scarring sequelae. However, the availability of donor sites that will enable the use of such grafts is limited, and thus, the application of split-thickness skin grafts becomes necessary ${ }^{[17,18]}$. Skin substitutes of human or animal origin or synthetic substitutes may be an alternative for reconstructing the dermis, reducing the incidence of retraction from the use of split-thickness skin grafts. These substitutes allow to compensate for the lack of donor sites of full-thickness skin grafts, bringing satisfactory results. Nonetheless, the limited availability of these dermal matrices and their elevated costs for most burn units in developing countries have hindered their use ${ }^{[19,20]}$.

Traction and tension forces on scars also play an important role during the formation of optimal scars. These forces are associated with the orientation of collagen fibers in the lower dermis and are essential for correcting hypertrophic scars, particularly when Z-plasties are performed to redirect scars into the relaxed skin tension lines, i.e., Langer's lines ${ }^{[21,22]}$. Nevertheless, Langer ${ }^{[21]}$ studied these relaxed skin tension lines in cadavers, whereas Kraissl's lines were defined in living individuals under no traumatic procedures ${ }^{[23]}$. In this way, Kraissl's lines are found to be more dynamic and to correlate with wrinkle lines during muscle contraction, making them more accurate than Langer's lines. These lines are disposed transversely, run perpendicular to muscle action, and they are vital in normal scarring ${ }^{[23,24]}$. In the light of the foregoing, we have suggested that placing split-thickness skin grafts transversely, following Kraissl's lines, on exposed areas resulting from burns may improve the esthetic and definitive functional outcomes in burn patients. This study aimed at describing patient evolution with split-thickness skin grafts applied by following Kraissl's lines during a 12-month period. To evaluate the functional and esthetic outcomes of scars in patients who underwent split-thickness skin grafting transversely based on Kraissl's lines to cover deep burns.

\section{METHODS}

A descriptive longitudinal case series study was conducted at the Burn Unit of Health Services (HSU) Simón Bolívar North Subnetwork E.S.E. of the Secretariat of Health in Bogotá, Colombia, from 1 Jan 2016 to 31 Dec 2017. The study was approved by the Institutional Ethics Committee, and was based on the ethical principles contained in the declaration of Helsinki.

The study included 138 patients with a mean age of 24.5 years (range: 7-74 years). Of this group, most subjects were men $(n=96,70 \%)$, with a mean age of 24.5 years (range: 7-74 years). These injuries required definitive coverage, involved any part of the body, with the exception of the face, and were classified as superficial 
(affecting only the epidermis), partial-thickness (until papillary dermis layer) or full-thickness (complete dermis and underlying tissue).

Patients were asked to sign an informed consent form to participate in the study and have their photos taken. Patients showing additional comorbidities that could affect scarring, including malnutrition, high blood pressure, diabetes mellitus, and coagulation disorders, were excluded. Patients having severe mental disorders or exposed areas not suitable for graft coverage, including hypertrophic granulation tissues, edematous or pale tissues, or the presence of discharge, were also excluded.

\section{Procedures}

Once the informed consent form was signed, the procedure was performed in operating rooms after the administration of general anesthesia, patients were washed with water and chlorhexidine soap. Granulation tissue curettage was executed for full removal, and wet compresses diluted in $500 \mathrm{cc}$ of $0.9 \%$ saline were applied, along with the use of a 1-mg adrenaline ampoule, to achieve hemostasis. In case bleeding was not controlled, hemostasis was ensured by electrocautery. Subsequently, from the available donor sites, such as the lower limbs, back or scalp, a split-thickness skin graft (between $0.25 \mathrm{~mm}$ and $0.30 \mathrm{~mm}$ ) was harvested using an electric dermatome. Following this, bupivacaine $0.25 \%$ with epinephrine at a dose of $2 \mathrm{mg} / \mathrm{kg}$ was administered to manage postoperative pain at donor site. Upon verifying hemostasis at receptor site, the graft was placed transversely in sheets, without expansion or meshing. Incisions for drainage were made, while ensuring that no exposed spaces were left in between each sheet. Suture immobilization remained at the discretion of the surgeon, but it was usually done on pediatric patients and at flexion creases sites. Next, the graft receptor site was covered with nitrofurazone-impregnated gauzes and cotton padding bandages as secondary dressing. Flexion areas were applied plaster splint intraoperatively. The first cleaning and dressing session took place at postoperative day 5, and from then on, every third day until hospital discharge. Postoperative monitoring varied in frequency, based on the surgeon's judgment, until postoperative month 12. The same two surgeons performed the monitoring.

On admission, all patients were evaluated and managed through physical and occupational therapies. Based on the reported findings, the rehabilitation team planned activities aimed at lowering edema, reducing pain, improving ROM, controlling positioning, and recovering sensitivity and strength, to promote the functionality of affected areas and independence of physical and instrumental ADL. Rehabilitation treatment was suspended immediately after surgery and was resumed at day 5 upon verification by the surgeon that there was proper graft integration. Once the physical and occupational therapies were resumed, they were continued during hospital admission and 3 months postoperatively (outpatient), through physical conditioning, digital pressure on the scar, orthotic management, Lycra garments, silicone, moisturizers, and lubricants. The scars had no medication infiltrated.

\section{Scarring evaluation}

The Vancouver Scar Scale (VSS) was used as follows: pigmentation and vascularity was examined visually, pliability was assessed by palpation and digital pressure, and height/thickness was measured with a millimeter ruler $^{[25]}$. Overall scores were categorized in three groups according to the following characteristics: (1) mild scar (score 0-3): characterized by a flat scar having a similar appearance to normal skin. It is supple and elastic, and has a few elements of pigmentation or vascularization; (2) moderate scar (score 4-7): characterized by a fairly thick and wide scar or a slightly wide and raised scar. Pink to red pigmentation changes may be observed, but there is yielding. Some areas may be fragile and less resistant; and (3) severe scar (score 8-13): characterized by clear signs of hypertrophy or keloid. The scar has a red to purple pigmentation, and it is unstable, raised, bulky, firm and indurated. Contractures and retractions may be present. Results were evaluated by a team of experts consisting of two surgeons, who performed the procedures, and a burn specialist. 
Transverse grafts in partial-thickness burns distribution by gender HSU Simón Bolívar North Subnetwork E.S.E. 2016-2017

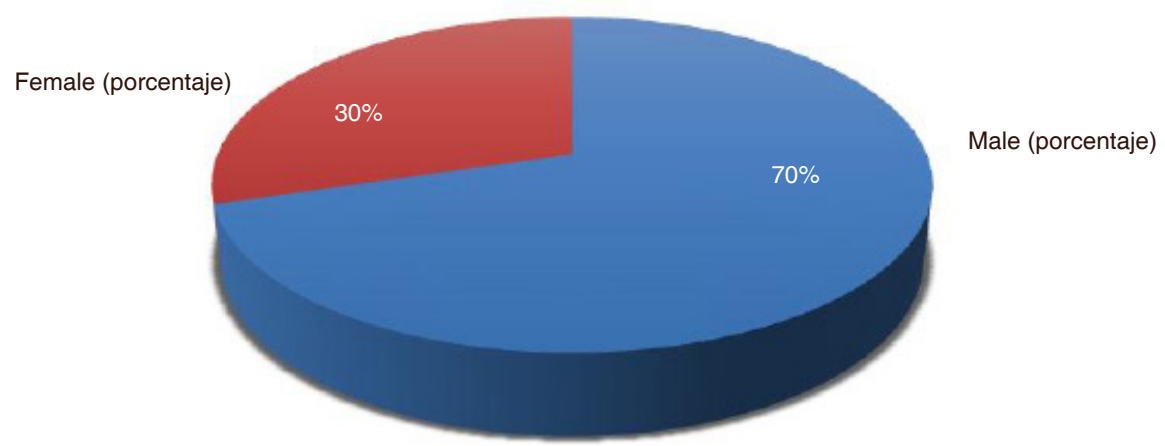

Figure 1. Distribution by gender of the 138 patients whose burns were treated with transverse split-thickness skin grafts. HSU: Unit of Health Services

Transverse grafts in partial - thickness burns distribution by age group HSU Simón Bolívar North Subnetwork 2016-2017

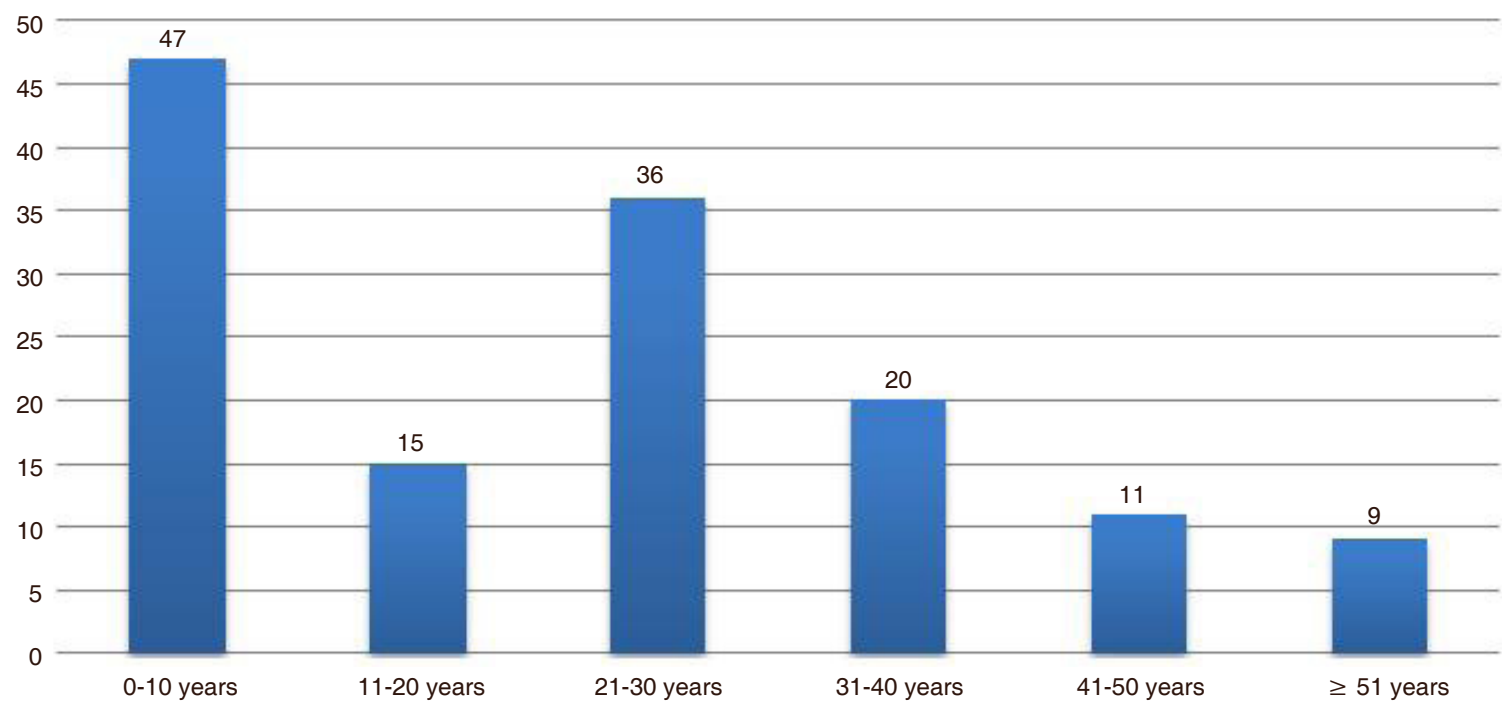

Figure 2. Distribution by age group. HSU: Unit of Health Services

\section{Statistical analysis}

The socio-demographic variables age and sex were registered. Other clinical characteristics were registered as well, including total burned body surface area using the Lund and Browder chart ${ }^{[26]}$, burn depth, compromised body area, eschar removal prior to applying definitive autografts, number of days since admission until definitive coverage, grafted anatomical area, donor sites, graft thickness, and complications. Scars were assessed at month 1 and at month 12 based on the VSS. For qualitative variables, frequencies and percentages were used, whereas for quantitative variables, mean, median and range were preferred. Microsoft Excel ${ }^{\circledR}$ was used.

\section{RESULTS}

A total of 138 patients showing deep burns were admitted. They required definitive coverage with splitthickness skin grafts, which were placed transversely. Of this group, most subjects were men [Figure 1], with a mean age of 24.5 years (range: 7-74 years) [Figure 2]. Among injury mechanisms, the most common etiologic factor was flame with $40 \%(n=55)$, followed by electricity $13.7 \%(n=19)$, scald $12.3 \%(n=17)$, chemical agents $13 \%(n=18)$, and other $21 \%(n=29)$ [Table 1$]$. 
Table 1. Socio-demographic variables and clinical characteristics of burns

\begin{tabular}{lcc}
\hline Variables & Frequency & Percentage \\
\hline Male & 96 & 70 \\
Female & 42 & 30 \\
Injury mechanism & & \\
Flame & 55 & 40 \\
Electricity & 19 & 13.7 \\
Scald & 17 & 12.3 \\
Deep partial-thickness burn & 52 & 37.7 \\
Full-thickness burn & 28 & 20.3 \\
Mixed burn (deep partial- and full-thickness) & 29 & 21 \\
& Median & Range \\
Age & 24.5 & $7-74$ \\
Burned body surface area & 24.34 & $2-66$ \\
\hline
\end{tabular}

The mean extension of total compromised body surface area was $24.34 \%$ (range: $2 \%-66 \%$ ). Concerning burn depth, 52 (37.7\%) patients exhibited partial-thickness burns, 28 (20.3\%) patients showed full-thickness burns, and $29(21 \%)$ patients had mixed burns, i.e., both deep partial- and full-thickness burns. The most common compromised anatomical areas were the upper limbs accounting for $77.4 \%(n=89)$, face $46 \%(n=53)$, trunk $40 \%(n=46)$, lower limbs 39.1\% $(n=45)$, and neck 21\% $(n=29)$. Mean hospital stay consisted of 16 days (range: 8-100 days).

Among the 138 patients, 106 (76.8\%) patients underwent a single early eschar removal procedure by tangential excision, 19 (13.7\%) patients required 2 escharectomies, 6 (4.3\%) patients 3 escharectomies, and 7 (5.0\%) patients 4 escharectomies. Patients having full-thickness burns underwent more escharectomies. In 26 (18.8\%) patients, the escharectomy area was covered temporarily with skin allografts prior to carrying out the definitive coverage with split-thickness skin autografts, as to promote the formation of granulation tissue suitable for grafts.

In all, $46(33.3 \%)$ patients required several grafting sessions, involving 2-5 additional procedures. These subjects exhibited burns $>20 \%$ of extension, full-thickness burns, and limited donor sites. Ten (7.2\%) of the 138 patients developed infection, resulting in full or partial graft failure. Reported cultures included Acinetobacter baumannii $(n=4,2.9 \%)$, Pseudomonas aeruginosa $(n=4,2.9 \%)$, and Klebsiella pneumoniae $(n=2,1.4 \%)$. No case of mortality was reported. Initial scars were assessed at first postoperative month, and definitive outcomes were determined at month 12 based on the VSS.

Of the 138 patients, 130 (94.2\%) showed mild scars (score $\leq 3$ in VSS). These were flat and had a similar appearance to normal skin; they were supple and elastic, and had a few elements of pigmentation (hypopigmentation and hyperpigmentation) or vascularization (pink) [Figures 3-6]. Four (2.9\%) patients showed moderate scars (score 4-7) having a $<5 \mathrm{~mm}$ thickness and width, and $\mathrm{a}<2 \mathrm{~mm}$ height. There were some changes in pigmentation and vascularization (pink to slight red); nonetheless, there was yielding and no evidence of rigidity or functional limitations, although some developed fragile areas with low resistance. Four (2.9\%) patients showed severe scars (score $\geq 8$ ) having clear signs of hypertrophy or keloid at 12-month followup. There was some degree of contracture and functional limitations, particularly in flexion creases sites. These $4(2.9 \%)$ patients developed infection in the grafted areas, and they required surgical correction through contracture release and the application of new grafts [Table 2].

In terms of rehabilitation, upon admission, all patients had developed some degree of edema in their upper and lower limbs. They also reported pain as per visual analog scale (VAS) at rest, which increased during muscle activity. Functional joint limitation was directly associated with skin involvement of finger and wrist 

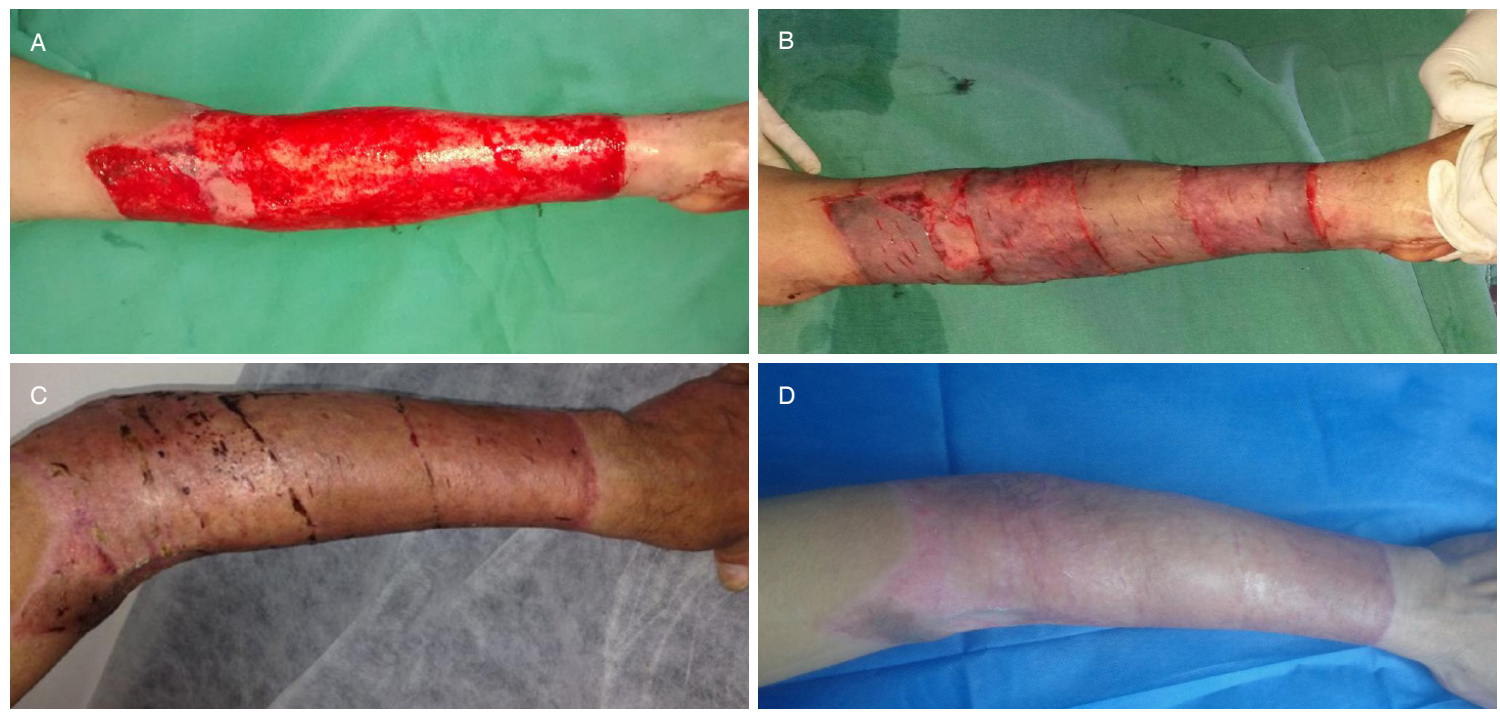

Figure 3. A 16-year-old female patient with a full-thickness flame burn to the left upper limb. A: Exposed area suitable for grafts; B: coverage with split-thickness skin grafts placed transversely; C: appearance by postoperative day 45; D: appearance after 6 months, with no functional limitations
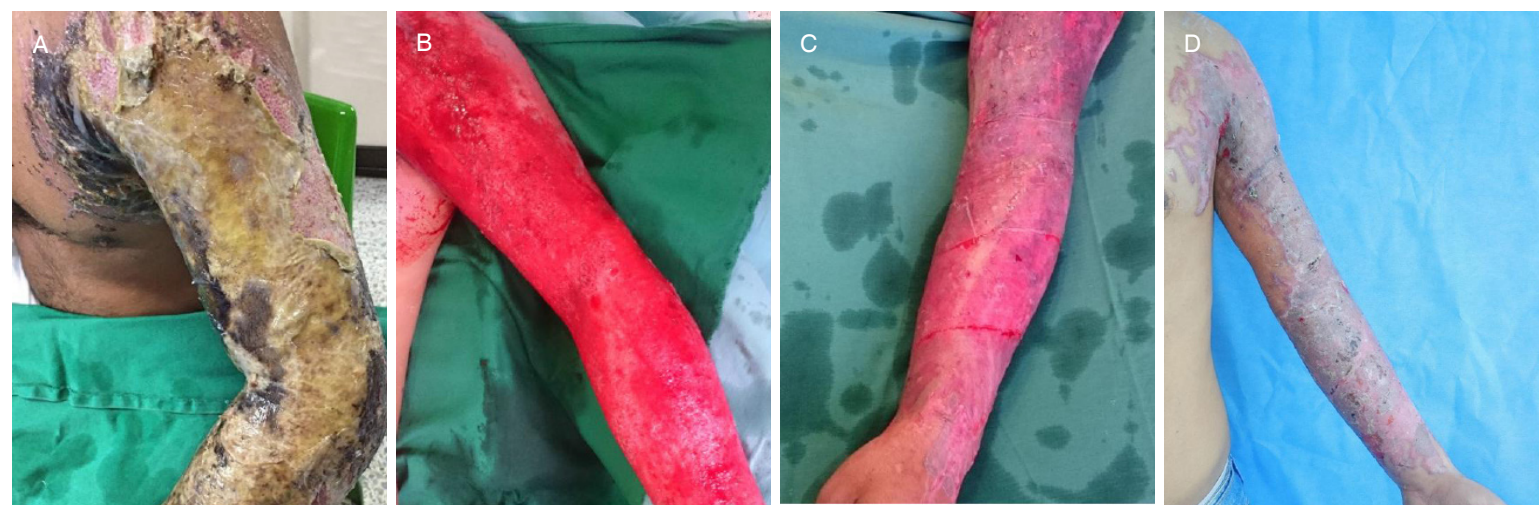

Figure 4. A 24-year-old male patient with a full-thickness chemical burn (sulfuric acid) to the left upper limb, involving creases. A: Initial appearance, with an adhered and hard eschar; B: formation of a uniform and non-hypertrophic granulation tissue. It was red and had no discharge following two escharectomies. It was covered temporarily with skin allografts; C: placement of split-thickness skin grafts transversely soon after surgery; D: appearance at postoperative month 1

joints, pain degree, and presence and severity of edema, resulting in functional limitation of the involved joint. Sensitivity exhibited mixed impairment, with areas of hyperesthesia associated with superficial burns and areas of hypoesthesia associated with deep burns, as well as non-painful areas corresponding to full-thickness burns. Patients showed decrease in strength, range of motion (ROM) impairment and activities of daily living (ADLs) limitations. This resulted in weakened or non-functional upper and lower limbs, making patients highly dependent.

At week 4 postoperatively, patients having a VSS score below 7 showed resolution of edema, a decrease to 0 for pain at rest and during activity (VAS), and gradual improvement of sensitivity during the following 6 months, as deep sensitivity was regained. There was an increase in strength, as well as greater gains in ROM and complete resumption of instrumental and non-instrumental ADLs. The 4 patients who developed infection and exhibited graft loss in affected areas showed scarring disorders, with signs of hypertrophic scars and keloids, cutaneous and subcutaneous contractures, and fascia contracture. None of them presented ligament 
Table 2. Classification of results based on the Vancouver Scar Scale

\begin{tabular}{lcc}
\hline Variable & Number of patients & Percentage \\
\hline Mild scar (Vancouver score $<3$ ) & 130 & $94.2 \%$ \\
Moderate scar (Vancouver score 4-7) & 4 & $2.89 \%$ \\
Severe scar (Vancouver score $\geq 8$ ) & 4 & $2.89 \%$ \\
\hline
\end{tabular}
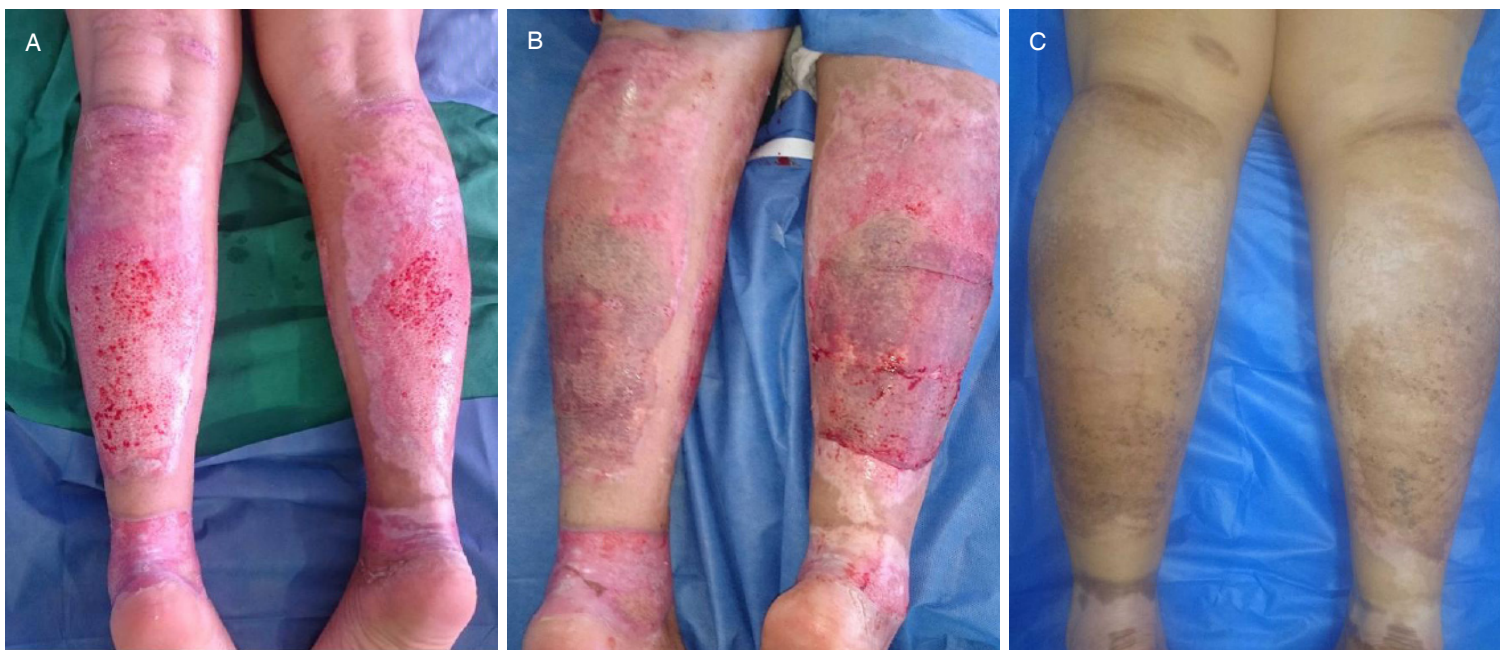

Figure 5. A: Thirty-two-year-old female patient with a deep partial-thickness flame burn to both lower limbs, who underwent eschar removal by tangential excision; B: placement of split-thickness skin grafts transversely; C: results at postoperative year 1. No evidence of contracture, and both texture and color are adequate
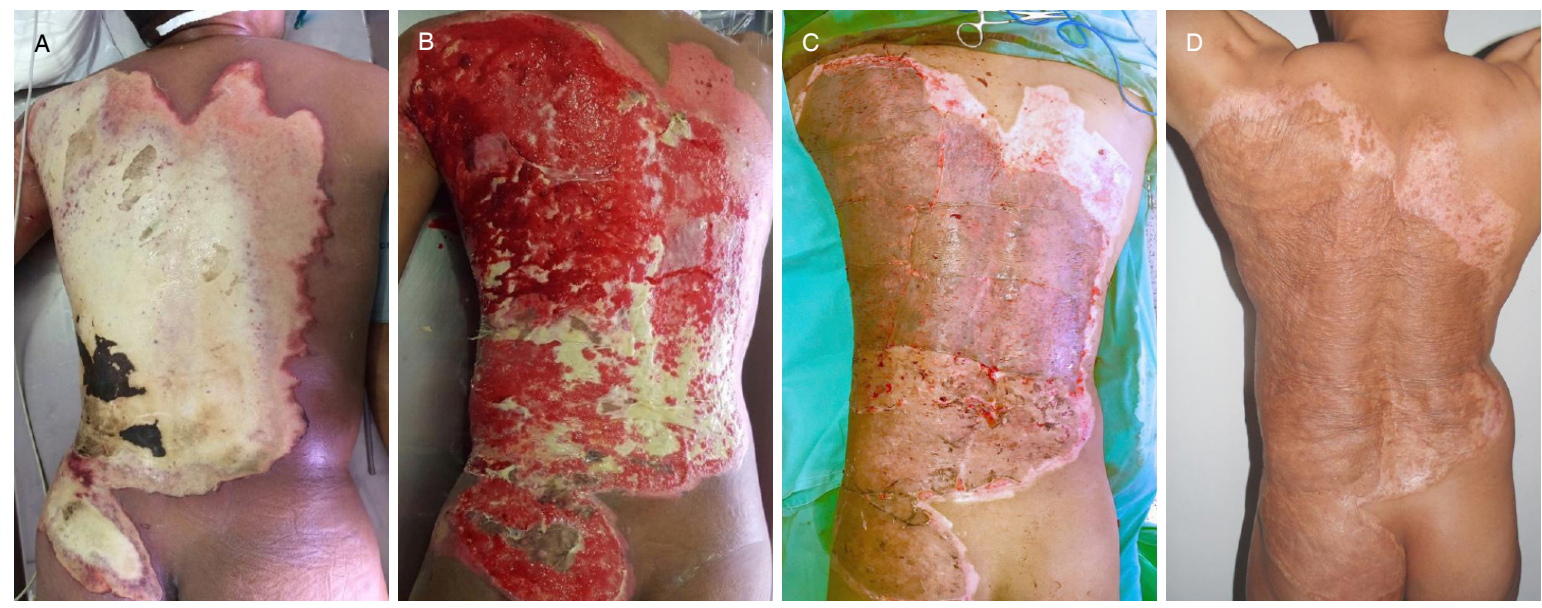

Figure 6. A: Twenty-eight-year-old male patient with a full-thickness flame burn of $18 \%$ total body surface area to back and gluteal region; B: appearance following 3 escharectomies performed by electric dermatome, and temporary coverage with skin allografts; C: intraoperative appearance after placing split-thickness skin grafts transversely; D: results at postoperative month 12

or muscle contractures, or functional limitations of involved joints; however, they reported persistence of pain at rest and during activity, decreased joint ROM, decrease in strength, and ADLs limitations, which improved at week 6 following surgical contracture release by placing new grafts and restarting rehabilitation.

\section{DISCUSSION}

In the researched literature, we did not find studies evaluating the evolution of split-thickness skin graft sheets in burn patients while taking into account their placement orientation. Conversely, abnormal scarring and 
joint contractures following deep burns have been widely reported. In a multicenter study involving 1865 patients, Goverman et al. ${ }^{[2]}$ described as risk factors and predictors of contracture development male sex, Hispanic ethnicity, medical problems, neuropathy, total body surface area (TBSA) affected by partial-thickness burns, TBSA grafted with split-thickness grafts, and prolonged hospital stay. According to their findings, the most common postburn morbidity was joint contracture, which occurred despite aggressive therapeutic interventions at both occupational and physical levels (positioning and splinting), affecting quality of life and ADLs. Their surgical management required skin grafts, as well as local and distant flaps. The most frequently contracted joint was the shoulder with $23.0 \%$, followed by the elbow (19.9\%), the ankle (13.6\%), and the knee (13.4\%). Schneider et al ${ }^{[4]}$ reported among 985 patients a joint contracture prevalence of $39 \%(n=381)$, with the shoulder, the elbow, and the knee being the most frequently contracted joints. In total, $39 \%$ of all cases developed at least one joint contracture. In 2003, Kowalske et al. ${ }^{[28]}$ in a study involving 1478 patients with major burns reported a joint contracture prevalence of $43 \%(n=641)$. Furthermore, in 2016 Oosterwijk et al. ${ }^{[29]}$ presented a systematic review according to which the prevalence of postburn contractures is different among studies, ranging from $38 \%$ to $54 \%$, and contractures were most likely to develop in deep and extensive burns, as well as in the neck and upper limbs.

In this study, we found that the transverse placement of grafts may lead to clear changes in terms of functionality and esthetics. The study sample revealed a very low incidence of contractures, adhesions, hypertrophic scars, and keloids (4/138, 2.9\%), particularly in limbs, which according to the literature constitute the most commonly affected areas by scarring sequelae. We consider that the transverse placement of grafts benefits scarring by reducing both tension of muscle contraction in grafted areas and resting skin tension, as the muscle action generated by Kraissl's lines correspond to the relaxed skin tension lines, which follow wrinkles and skin movement during muscle contraction, mainly in the neck, trunk, and limbs. In this way, such lines should be considered during the planning and placement of grafts. Unlike Goverman et al. . $^{[27}$, we believe that a multidisciplinary treatment with rehabilitation upon patient admission greatly influences prevention control of functional and scarring disorders. These observations are preliminary, and therefore, not conclusive, as they require further studies allowing comparisons with a control group.

Nevertheless, surgeons usually determine how to place split-thickness skin grafts on exposed areas, relying on the orientation that will allow the operator to cover the greatest exposed tissue area with the obtained graft. In this sense, the orientation of those forces resulting in the underlying muscle tissue contraction of the area to be grafted are not taken into consideration. Generally, the skin is under constant tension, which varies depending on the area. This static tension follows predictable patterns, and it is defined by Kraissl's lines. The movement of joints and muscles causes dynamic tension, and so placing grafts perpendicular to Kraissl's lines creates more tension, resulting in wider and hypertrophic scars that may even progress to severe contractures and functional impairments, especially in the limbs and neck.

Infection processes in burn patients continue to be a great public health problem associated primarily with an increase in mortality and morbidity, hospital stay, procedures, and costs. In our study, the occurrence of infection caused full or partial graft failure, thereby increasing the number of surgical procedures and prolonging hospital stay, as exposed areas required new grafts. Four of these patients obtained a score of $\geq 8$ in the VSS, attributable to a more active inflammatory process, increased fibrosis, prompted formation of hypertrophic scars, and some degree of contracture with functional limitations of the limbs. This sample includes patients of all ages, ranging from 1 to 80 years, having extensions of 3-66. Nevertheless, results did not vary based on age or extension.

Although not part of this study's objectives, we noted that placing grafts transversely enables their rationalization, since they are applied from both edges at the exact size of the defect, preventing the loss 
of small skin fragments. Conversely, placing grafts without considering their orientation usually involves the cutting of skin segments that protrude from the wound, which results in the loss of multiple small skin fragments.

Based on the results obtained in this study, we believe that tension forces acting on the grafted areas are essential in scarring. Orientation seems to be a relevant factor for such forces to exert more or less tension on the scar. Orienting grafts transversely, following Kraissl's lines, lowers tension of these scarring forces, which translates into more esthetic and functional characteristics. Further studies are required engaging a larger population sample and having multicenter and controlled designs that will allow to determine and measure the static and dynamic forces involved in the scarring of skin grafts.

In conclusion, burns are a type of trauma resulting in great morbidity rates. They compromise large portions of the skin in each patient and require a multidisciplinary and multimodal management to improve functionality and scar appearance. Abnormal scars are caused by several factors that affect scarring. One of the primary factors involves the tension exerted on the wound. Considering these relaxed skin tension lines, when applying split-thickness skin graft sheets transversely, reduces tension and minimizes the risk of developing abnormal scars, thereby achieving better esthetic results.

\section{DECLARATIONS}

\section{Authors' contributions}

Concept and design: Madiedo R

Data acquisition: Gaviria-Castellanos JL

Data analysis, manuscript preparation: Gaviria-Castellanos JL, Zapata-Ospina A

Critical revision and completion of manuscript: Madiedo R, Gaviria-Castellanos JL

\section{Availability of data and materials}

Data were strictly obtained from medical records, in accordance with the privacy policy and code of ethics of our institute.

\section{Financial support and sponsorship}

None.

\section{Conflicts of interest}

All authors declared that there are no conflicts of interest.

\section{Ethical approval and consent to participate}

This study was approved by the Hospital and all authors gave their consent to participate. Ethical approval number UI-19-15.

\section{Consent for publication}

Not applicable.

\section{Copyright}

(c) The Author(s) 2018.

\section{REFERENCES}

1. Smolle C, Cambiaso-Daniel J, Forbes AA, Wurzer P, Hundeshagen G, Branski LK, Huss F, Kamolz LP. Recent trends in burn epidemiology worldwide: a systematic review. Burns 2017;43:249-57. 
2. Wasiak J, McMahon M, Danilla S, Spinks A, Cleland H, Gabbe B. Measuring common outcome measures and their concepts using the international classification of functioning, disability and health (ICF) in adults with burn injury: a systematic review. Burns 2011;37:91324.

3. Ryan CM, Schoenfeld DA, Thorpe WP, Sheridan RL, Cassem EH, Tompkins RG. Objective estimates of the probability of death from burn injuries. N Engl J Med 1998;338:362-6.

4. Schneider JC, Holavanahalli R, Helm P, Goldstein R, Kowalske K. Contractures in burn injury: defining the problem. J Burn Care Res 2006;27:508-14.

5. Shichinohe R, Yamamoto Y, Kawashima K, Kimura C, Ono K, Horiuchi K, Yoshida T, Murao N, Hayashi T, Funayama E, Oyama A, Furukawa H. Factors that affected functional outcome after a delayed excision and split-thickness skin graft on the dorsal side of burned hands. J Burn Care Res 2017;38:e851-8.

6. Hop MJ, Langenberg LC, Hiddingh J, Stekelenburg CM, van der Wal MB, Hoogewerf CJ, van Koppen ML, Polinder S, van Zuijlen PP, van Baar ME, Middelkoop E. Reconstructive surgery after burns: a 10-year follow-up study. Burns 2014;40:1544-51.

7. Ladak A, Tredget EE. Pathophysiology and management of the burn scar. Clin Plast Surg 2009;36:661-74.

8. Ahn CS, Maitz PK. The true cost of burn. Burns 2012;38:967-74.

9. Hop MJ, Polinder S, van der Vlies CH, Middelkoop E, van Baar ME. Costs of burn care: a systematic review. Wound Repair Regen 2014;22:436-50.

10. Tenenhaus M, Rennekampff HO. Current concepts in tissue engineering: skin and wound. Plast Reconstr Surg 2016;138:42S-50S.

11. Shakespeare P, Shakespeare V. Survey: use of skin substitute materials in UK burn treatment centres. Burns 2002;28:295-7.

12. Khoo TL, Halim AS, Saad AZ, Dorai AA. The application of glycerol-preserved skin allograft in the treatment of burn injuries: an analysis based on indications. Burns 2010;36:897-904.

13. Wainwright DJ. Burn reconstruction: the problems, the techniques, and the applications. Clin Plast Surg 2009;36:687-700.

14. Khansa I, Harrison B, Janis JE. Evidence-based scar management: how to improve results with technique and technology. Plast Reconstr Surg 2016;138:165S-78S.

15. Esselman PC. Burn rehabilitation: an overview. Arch Phys Med Rehabil 2007;88:S3-6.

16. Simons M, King S, Edgar D; ANZBA. Occupational therapy and physiotherapy for the patient with burns: principles and management guidelines. J Burn Care Rehabil 2003;24:323-35.

17. Hazani R, Whitney R, Wilhelmi BJ. Optimizing aesthetic results in skin grafting. Am Surg 2012;78:151-4.

18. Jewell L, Guerrero R, Quesada AR, Chan LS, Garner WL. Rate of healing in skin-grafted burn wounds. Plast Reconstr Surg 2007;120:4516.

19. Sheridan R. Closure of the excised burn wound: autografts, semipermanent skin substitutes, and permanent skin substitutes. Clin Plast Surg 2009;36:643-51.

20. Kagan RJ, Peck MD, Ahrenholz DH, Hickerson WL, Holmes J 4th, Korentager R, Kraatz J, Pollock K, Kotoski G. Surgical management of the burn wound and use of skin substitutes: an expert panel white paper. J Burn Care Res 2013;34:e60-79.

21. Langer K. On the anatomy and physiology of the skin: I. the cleavability of the cutis. Br J Plast Surg 1978;31:3-8.

22. Lemperle G, Tenenhaus M, Knapp D, Lemperle SM. The direction of optimal skin incisions derived from striae distensae. Plast Reconstr Surg 2014;134:1424-34.

23. Kraissl CJ. The selection of appropriate lines for elective surgical incisions. Plast Reconstr Surg (1946) 1951;8:1-28.

24. Converse JM, Robb-Smith AH. The healing of surface cutaneous wounds: its analogy with the healing of superficial burns. Ann Surg 1944;120:873-85.

25. Sullivan T, Smith J, Kermode J, McIver E, Courtemanche DJ. Rating the burn scar. J Burn Care Rehabil 1990;11:256-60.

26. Lund CC, Browder NC. The estimation of areas of burns. Surg Gynecol Obste 1944;79:352-8.

27. Goverman J, Mathews K, Goldstein R, Holavanahalli R, Kowalske K, Esselman P, Gibran N, Suman O, Herndon D, Ryan CM, Schneider JC. Pediatric contractures in burn injury: a burn model system national database study. J Burn Care Res 2017;38:e192-9.

28. Kowalske K, Holavanahalli R, Serghio M, Esselman P, Ware L, Delateur B, Helm P. Contractures following burn injury in children and adults - a multicenter report. J Burn Care Res 2003;24:S85.

29. Oosterwijk AM, Mouton LJ, Schouten H, Disseldorp LM, van der Schans CP, y Nieuwenhuis MK. Prevalence of scar contractures after burn: a systematic review. Burns 2017;43:41-9. 